\title{
Efficacy and safety of radio-chemotherapy combined with thermotherapy for advanced cervical cancer in Chinese women: a meta-analysis
}

\author{
Qi Baoning, Shi Chuandao, Sun Na, Wang Xinan, Han Yuanping
}

Department of Public Health and Prevention, Shaanxi University of Chinese Medicine, Xianyang, China

Submitted: 22 October 2017

Accepted: 12 December 2017

Arch Med Sci Civil Dis 2017; 2: e182-e190

DOI: https://doi.org/10.5114/amscd.2017.72542

Copyright $@ 2017$ Termedia \& Banach

\section{Abstract}

Introduction: Pelvic deep hyperthermia is an important treatment method for cervical cancer, and it has been widely used in Chinese clinics. However, this approach has not been widely adopted all over the world because of its uncertain efficacy and safety. This meta-analysis aims to review the efficacy and safety of radio-chemotherapy combined with local hyperthermia $(\mathrm{HT})$ in the treatment of cervical cancer.

Material and methods: We searched foreign and domestic databases, and also searched the related references for additional information. Data extraction and quality assessment were conducted by two reviewers independently. Rev Man 5.3 software was used to perform the meta-analysis.

Results: The thermotherapy group had a significantly higher 1-year tumor-free survival rate $(\mathrm{OR}=2.84,95 \% \mathrm{Cl}(1.14-7.09), p=0.03)$, complete remission rate $(\mathrm{OR}=2.41,95 \% \mathrm{Cl}(1.94-3.01), p<0.00001)$ and overall effective rate $(\mathrm{OR}=4.11,95 \% \mathrm{Cl}(3.11-5.44), p<0.00001)$ than the sole radio-chemotherapy group. The thermotherapy group had a significantly lower disease stability rate $(\mathrm{OR}=0.44,95 \% \mathrm{Cl}(0.32-0.62), p<0.00001)$ and disease progression rate $(\mathrm{OR}=0.15,95 \% \mathrm{Cl}(0.08-0.28), p<0.00001)$ when compared with the sole radio-chemotherapy group. Both groups were statistically similar with respect to adverse reactions.

Conclusions: Radio-chemotherapy combined with thermotherapy could significantly improve short-term curative effects for patients with cervical cancer. However, due to the limited quantity and quality of the included studies, more high-quality studies with a large sample size and long-term follow-up are still needed to verify the above conclusion and explore its long-term efficacy.

Key words: cervical cancer, thermotherapy, radio-chemotherapy, efficacy, meta-analysis.

\section{Introduction}

Cervical cancer is one of the most common gynecological malignancies, and also a serious threat to female physical and mental health. There are about 528,000 new cases of cervical cancer every year in the world, of which about 27,000 die of this disease [1]. Currently, combination of radiotherapy with chemotherapy is widely used and also acts as a standard treatment with excellent local control and low toxicity [2, 3]. However, its long-term survival rate has still not reached the intend-

\author{
Corresponding author: \\ Qi Baoning \\ Department of Public \\ Health and Prevention \\ Shaanxi University \\ of Chinese Medicine \\ Century Avenue \\ 712046 Xianyang \\ Shaanxi Province \\ 712046 Xianyang, China \\ Phone: +86 15877402750 \\ E-mail: qbn0506@stu.xjtu. \\ edu.cn
}


ed effect. Studies in the early 1990s had already explored the effectiveness of hyperthermia as an adjuvant therapy for radiotherapy or chemotherapy in treating advanced cervical cancer [4]. Recently, locally pelvic deep hyperthermia as an important treatment for cervical cancer has been widely used in clinics, its therapeutic effect has gained great recognition [5-7], and it has already become a conventional adjuvant therapy for cervical cancer treatment in China. However, the randomized controlled trials (RCTs) are mostly small sample size and single-center studies with much lower reliability, thus hindering the thermotherapy widely used in clinical practice all over the world. Therefore, in this meta-analysis, we systematically evaluated the efficacy and safety of local hyperthermia combined with radio-chemotherapy in treating cervical cancer to provide a more reliable basis in clinical application and research.

\section{Material and methods}

\section{Literature inclusion and exclusion criteria}

(1) Studies are randomized controlled trials (RCTs). (2) Studies focused on patients with cervical cancer and without radio-chemotherapy and thermotherapy contraindications, and the age of patients is not limited. (3) Comparative studies were between radio-chemotherapy (CRT) and CRT + local hyperthermia (HT). (4) Efficacy determination was conducted according to the solid tumor efficacy evaluation criteria. Outcome index included: a. long-term efficacy (3-year survival rate, 3-year SR); b. short-term efficacy (1year tumor-free survival rate, 1-year TFSR), complete remission rate (CRR), partial remission rate (PRR), overall effective rate (OER); disease stability rate (SDR); disease progression rate (PDR); $c$. the acute-care side effect including gastrointestinal reaction, bone marrow suppression and bladder reaction. (5) Studies with sample size $<50$ cases, repeat publications, non-original studies, animal tests or preclinical trials, and abstract-only publications were excluded.

\section{Search strategy}

We searched for studies comparing HCRT with CRT for the treatment of cervical cancer using Cochrane Library, PubMed and Embase, along with four domestic databases (CBM, VIP, CNKI, and Wan Fang Data). Retrieval time was from 1 January 2000 to 31 December 2016. The combination of subject search with free word examination approach was adopted for retrieval. The search terms were: "cervical cancer", "uterine neoplasms/cancer”, "gynecologic cancer", "radiotherapy", "chemotherapy", "drug therapy", "thermotherapy", "hyperthermia”, "fever", "heat and randomized controlled trials".
The literature was incorporated on radiotherapy and chemotherapy combined with hyperthermia in the treatment of advanced cervical cancer, and the relevant literature was also searched on the Internet.

\section{Data extraction and quality assessment}

Two reviewers independently extracted the safety and efficacy indexes into a prospective data extraction form and checked them twice. Disagreements were resolved by discussing or consulting the third author. The methodological quality of the included studies was assessed using the RCT risk-based assessment tool based on the Cochrane System Reviewer's Manual 5.3. The main contents of the data extraction included the first author, publication date, grouping method, Karnofsky score, FIGO stage, pathological type, radiotherapy, and chemotherapy. The outcomes such as survival rate, complete remission rate, partial remission rate, overall effective rate, and the related incidence of adverse reactions were extracted. According to the RECIST (Provide Evaluation Criteria in Solid Tumors) definition, complete remission (CR) means all lesions disappeared completely and no new lesions appeared, and tumor markers dropped to normal levels for 4 weeks. Partial remission (PR) means that the largest diameter of the tumor decreased by $>30 \%$, and was maintained for 4 weeks. Stabilization (SD): lesions increased less than in PD, and decreased less than in PR. Progress (PD): lesion diameter increased $>20 \%$, or the emergence of new lesions. The total effective rate or overall effective rate is $(O R)=$ $(C R+P R) /$ total number of cases $\times 100 \%$.

\section{Statistical analysis}

The meta-analysis was conducted using Review Manager 5.3 (RevMan, The Cochrane Collaboration, Oxford, UK). The Cochrane-Q statistic and the $I^{2}$ statistic were used to assess statistical heterogeneity. The $\chi^{2}$ test was used to analyze the heterogeneity of all the included studies. Inspection level is $\alpha=0.1$, and $p$-value $\geq 0.10$ and $I^{2}$ values $\leq 50 \%$ illustrated that the selected studies were consistent with homogeneity, which was suitable for a fixed-effect model for meta-analysis; If $p$-value $<0.10$ as well as $l^{2}$ values $<50 \%$, it showed that heterogeneity existed among the studies but within an acceptable range, which also used the fixed-effect model meta-analysis; When the $p$-value $<0.10$ and $R$ values $>50 \%$, it indicated greater heterogeneity among the results, which necessitated further analysis of its sources of heterogeneity. If without obvious clinical heterogeneity, the random-effect model can be used for analysis; if heterogeneity is extremely obvious, descriptive analysis was chosen. Odds ratios (ORs) 
and $95 \%$ confidence intervals (Cls) were used as the summary variables for final outcomes.

\section{Results}

\section{Description of selected studies and quality assessment}

A total of 471 articles were related to the initial examination. After reading the abstracts as well as the full texts, irrelevant or non-comparative studies, reviews, abstracts, case reports, repeated and narrative studies, small sample studies, non-RCTs and seriously missing data studies were excluded, and after the first meta-analysis, papers of low quality were removed. Finally, 19 RCTs were included in the study for further analysis. The flow diagram for study selection is shown in Figure 1. The characteristics of the included studies are summarized in Table I [8-26]. Although some missing data were not adequately described in some studies, the results showed that the basic information between the test group and the control group had good comparability, which suggested that the investigations included in this study were of high quality.

\section{Short-term efficacy}

For 1-year tumor-free survival rate (1-year TFSR), five studies with a total of 615 patients were included in this analysis. When compared with the CRT group, the HCRT (hyperthermia and radio-chemotherapy) group had significantly im- proved 1-year TFSR (OR $=2.84,95 \% \mathrm{Cl}: 1.14-7.09$ $p=0.03$ ) (Figure $2 \mathrm{~A}$ ). Eighteen studies described the CRR, and the fixed-effects model of meta-analysis showed that CRR was significantly higher in the HCRT group than in the CRT group $(\mathrm{OR}=2.41,95 \% \mathrm{Cl}: 1.94-3.01, p<0.00001)$ (Figure $2 \mathrm{~B}$ ). The partial remission rates (PRR) of both CRT and HCRT groups were statistically similar $(\mathrm{OR}=0.92,95 \% \mathrm{Cl}: 0.72-1.17)$ (Figure $2 \mathrm{C})$. The benefit to the OER was significantly higher in the HCRT group than in the CRT group (OR $=4.11,95 \%$ $\mathrm{Cl}: 3.11-5.44, p<0.00001)$ (Figure 3). Additionally, the HCRT group had a significantly lower SDR (OR $=0.44,95 \% \mathrm{Cl}: 0.32-0.62, p<0.00001$ ) (Figure $4 \mathrm{~A})$ and PDR (OR $=0.15,95 \% \mathrm{Cl}: 0.08-0.28, p<$ 0.00001) (Figure 4 B).

\section{Long-term efficacy}

The long-term efficacy was evaluated by the 3-year survival rate at the end of the treatment. Only three of the included studies compared the 3-year survival rate between the HCRT group and the CRT group, and the meta-analysis showed that the 3-year survival rate of the HCRT group was similar to that of the CRT group, without statistical significance $(\mathrm{OR}=1.17,95 \% \mathrm{Cl}: 0.80-1.72, p=$ 0.42) (Figure 5).

\section{Adverse effects in different groups}

Fourteen studies reported the incidences for gastrointestinal disorders and the bone marrow

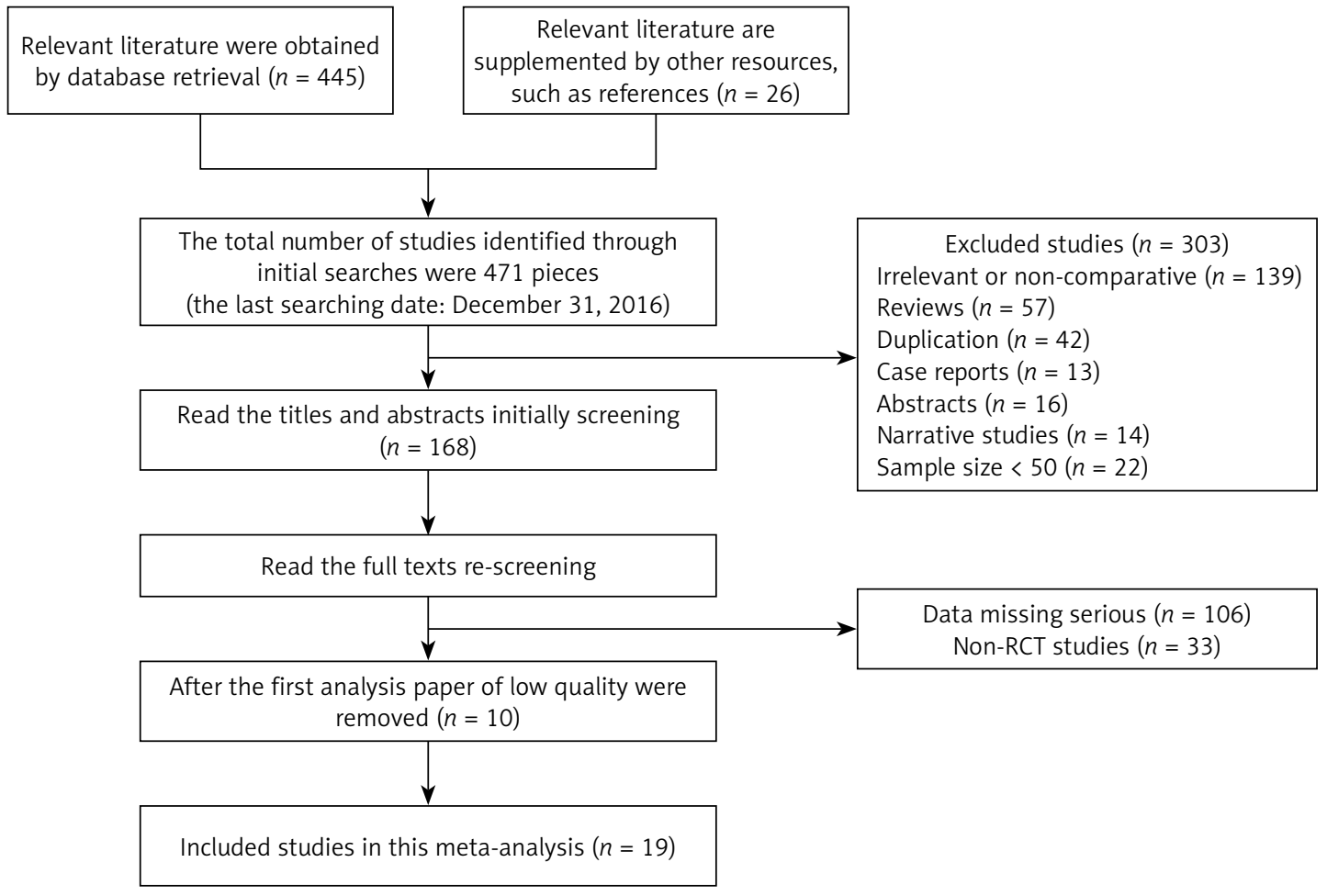

Figure 1. Flow diagram of the literature search and study selection 


\begin{tabular}{|c|c|c|c|c|c|c|c|c|c|c|c|c|c|c|c|c|c|c|c|}
\hline 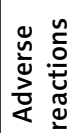 & \begin{tabular}{|c|}
$\bar{\Xi}$ \\
$\frac{\tilde{J}}{\breve{J}}$ \\
$\stackrel{J}{J}$
\end{tabular} & 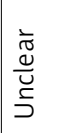 & 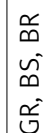 & 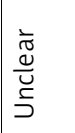 & 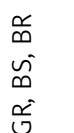 & 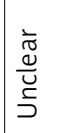 & 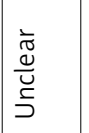 & $\begin{array}{l}\frac{\alpha}{\infty} \\
w \\
\infty \\
\frac{\alpha}{0}\end{array}$ & $\begin{array}{l}\text { or } \\
\infty \\
w \\
\infty \\
\text { s. }\end{array}$ & 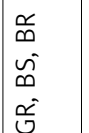 & $\begin{array}{l}\infty \\
\infty \\
\sim \\
\infty \\
\alpha-j\end{array}$ & 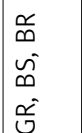 & $\begin{array}{l}\tilde{\alpha} \\
\tilde{u} \\
\tilde{u}\end{array}$ & 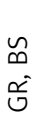 & $\begin{array}{l}\widetilde{\alpha} \\
\tilde{s}\end{array}$ & $\begin{array}{l}\widetilde{m} \\
\stackrel{s}{\circlearrowleft}\end{array}$ & $\begin{array}{l}\stackrel{\alpha}{\infty} \\
\text { s. } \\
\text { s. }\end{array}$ & 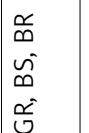 & \\
\hline 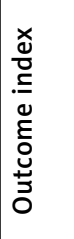 & 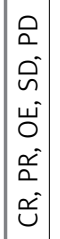 & 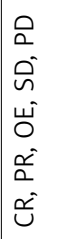 & 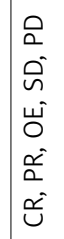 & $\begin{array}{l}\tilde{v} \\
m \\
\tilde{v} \\
\tilde{v} \\
\tilde{u} \\
0\end{array}$ & 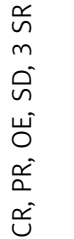 & $\begin{array}{l}0 \\
a \\
0 \\
u \\
0 \\
\check{a} \\
a \\
\tilde{u}\end{array}$ & 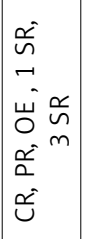 & 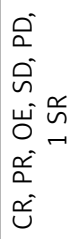 & 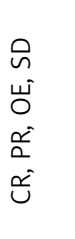 & 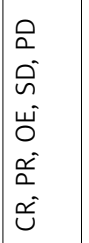 & 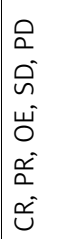 & 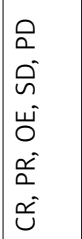 & 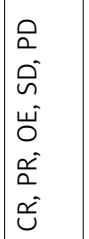 & 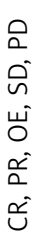 & 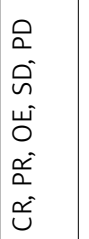 & 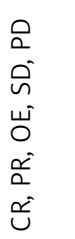 & 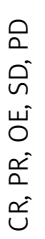 & 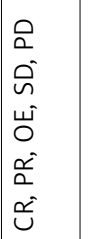 & \\
\hline 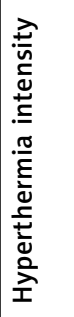 & 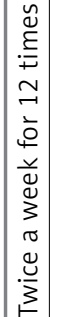 & 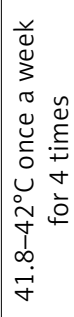 & 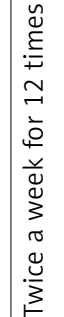 & 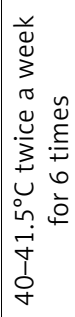 & 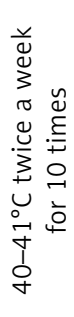 & 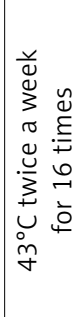 & 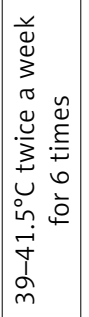 & 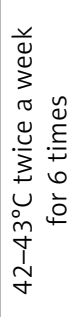 & 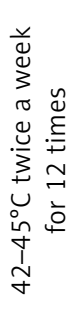 & 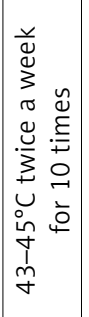 & 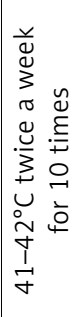 & 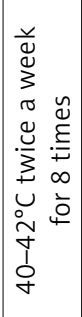 & 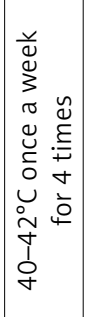 & 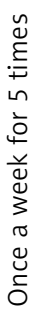 & 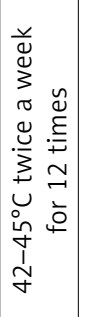 & 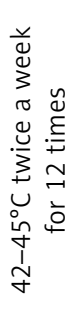 & 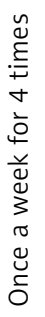 & 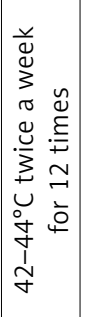 & \\
\hline 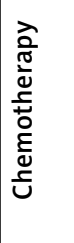 & 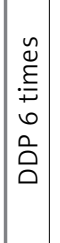 & 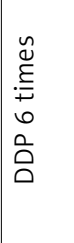 & 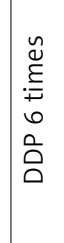 & 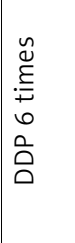 & 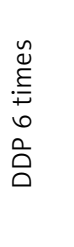 & 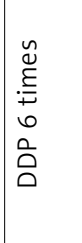 & 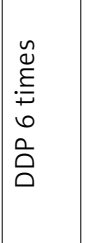 & 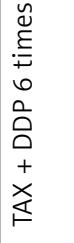 & 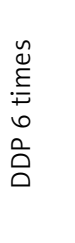 & 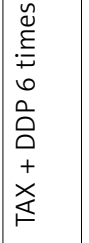 & 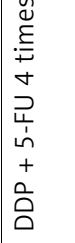 & 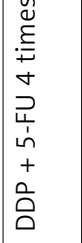 & 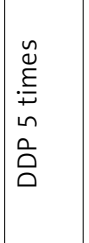 & 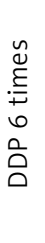 & 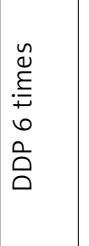 & 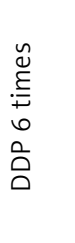 & $\begin{array}{l}\stackrel{\tilde{\omega}}{\underline{\xi}} \\
0 \\
0\end{array}$ & 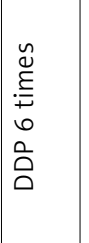 & \\
\hline
\end{tabular}

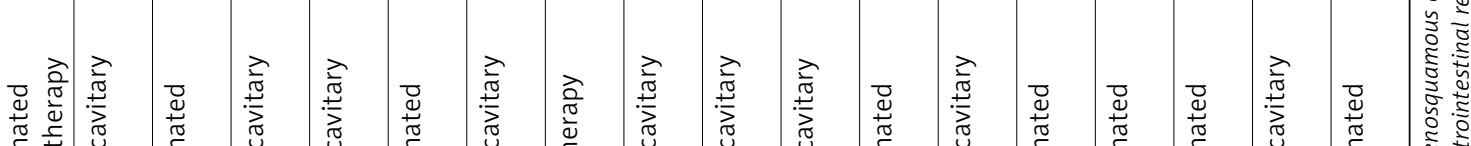

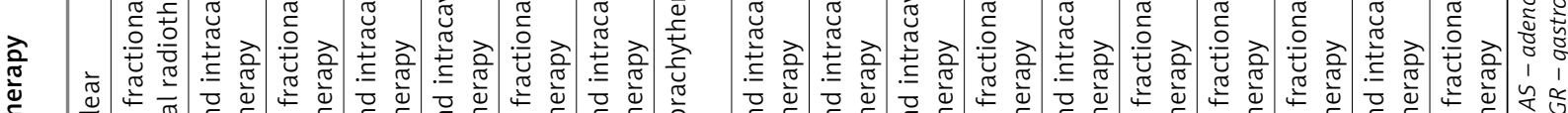

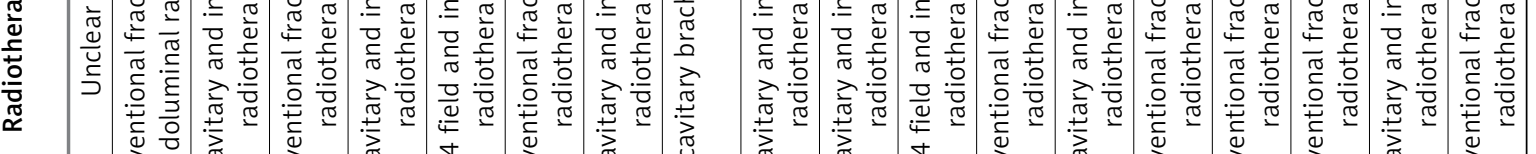

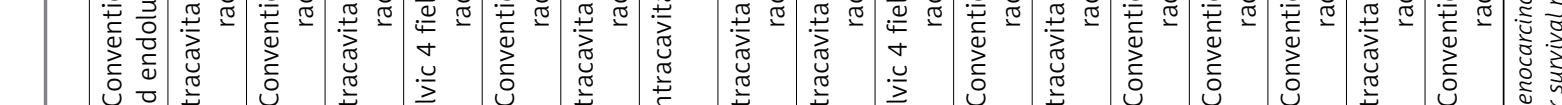

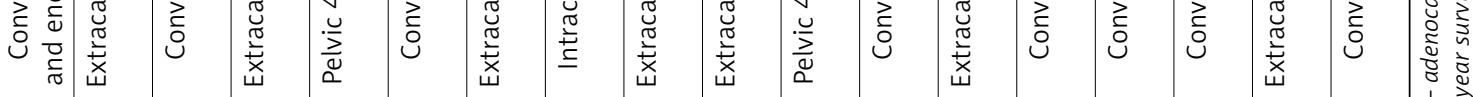

ए人

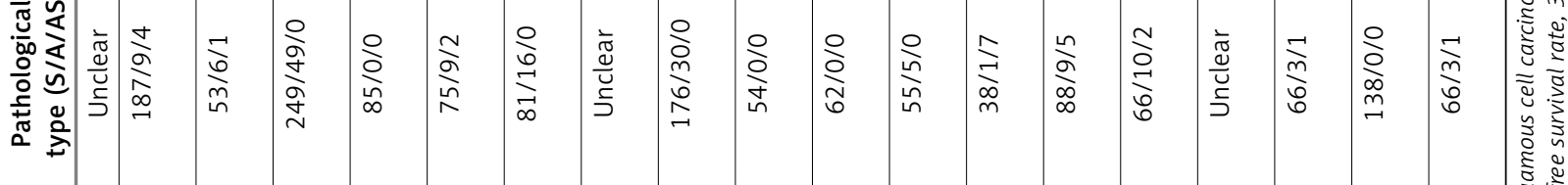

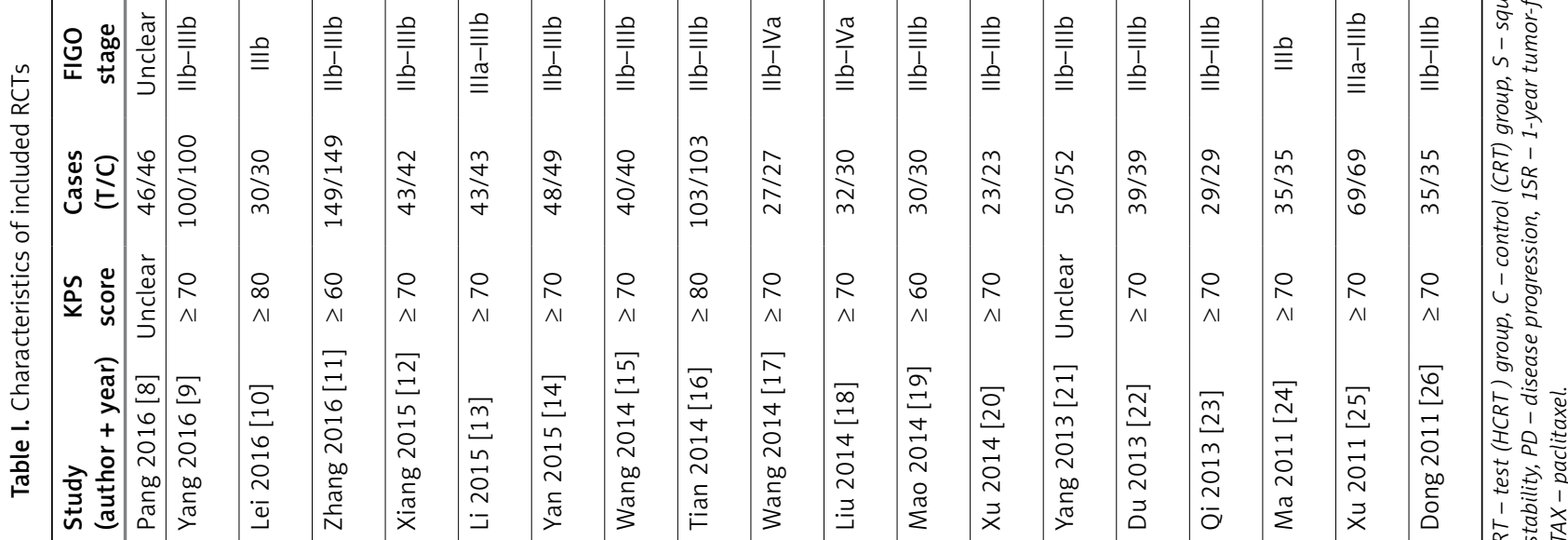


A

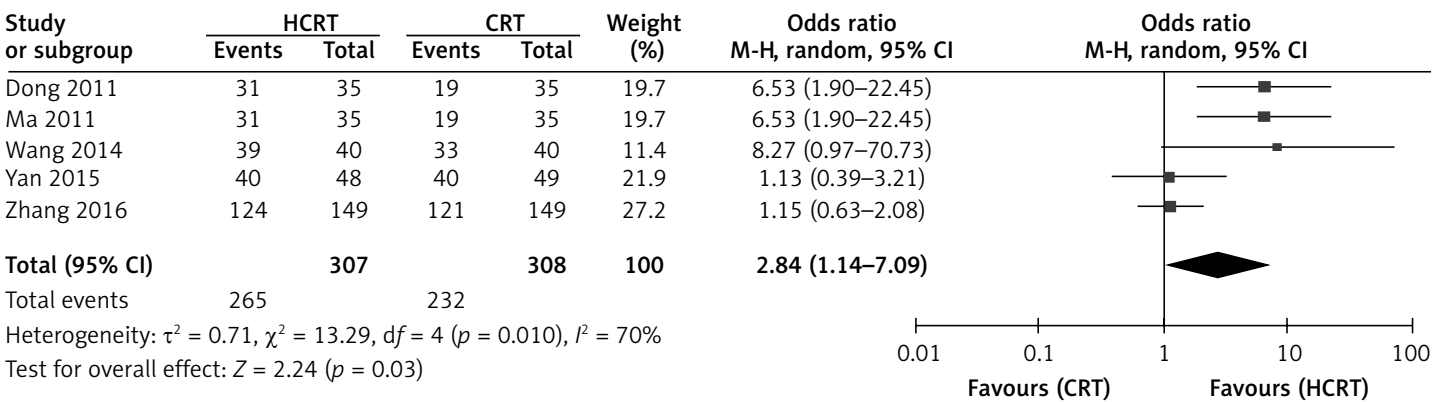

B

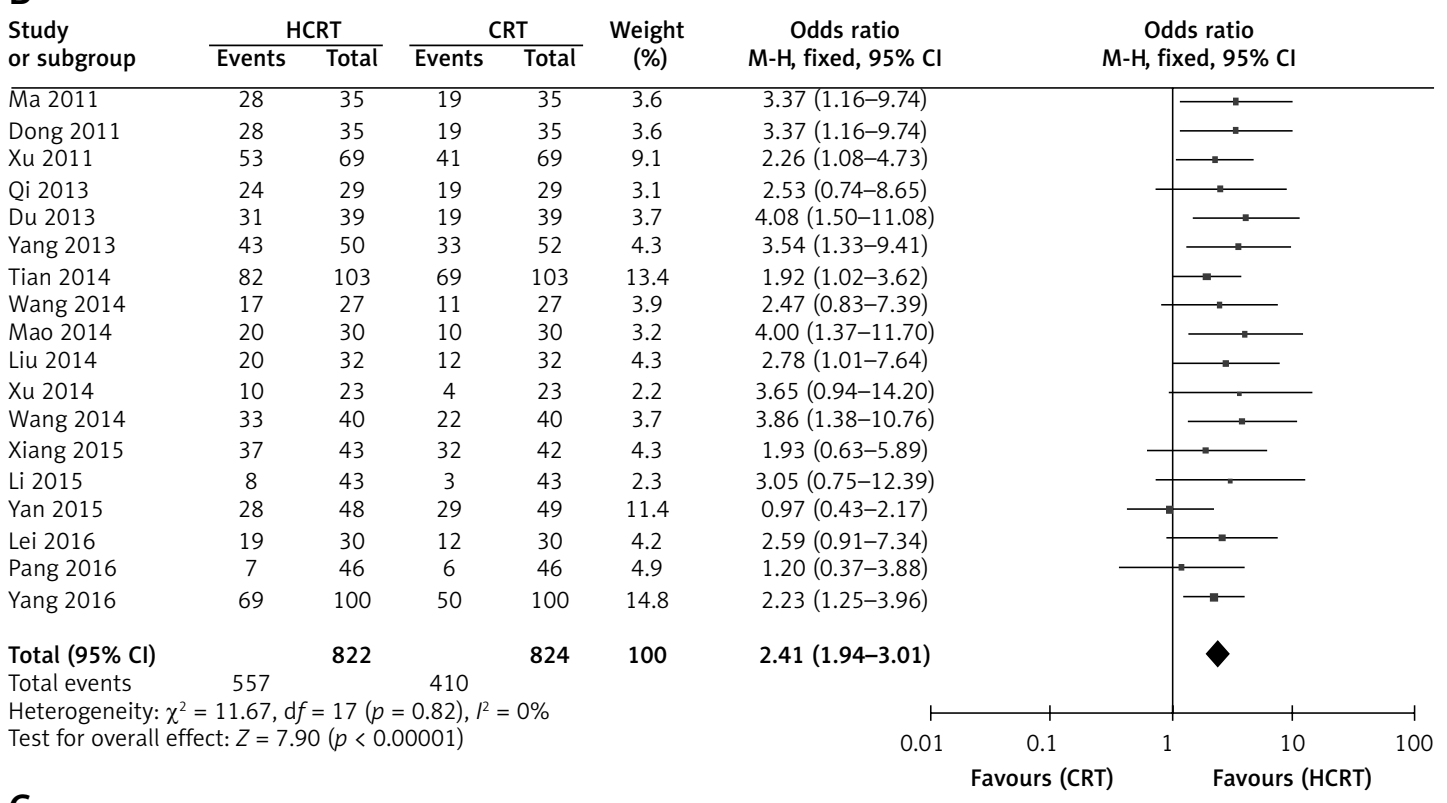

C

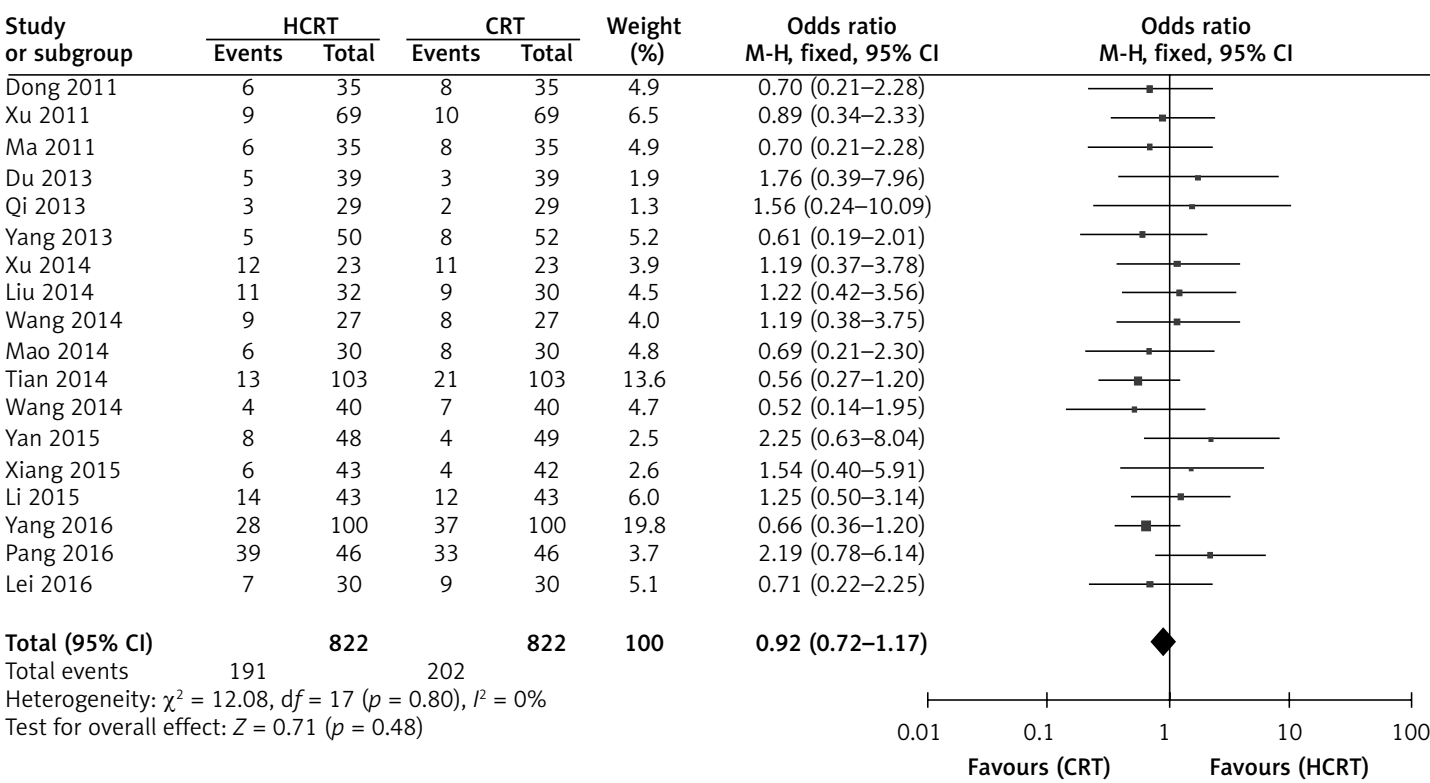

Figure 2. Forest plot for meta-analysis of the short-term efficacy of 1-year tumor-free survival rate (1-year TFSR $(A))$, complete remission rate (CRR (B)), and progression rate (PRR (C)) between the CRT group and the HCRT group 


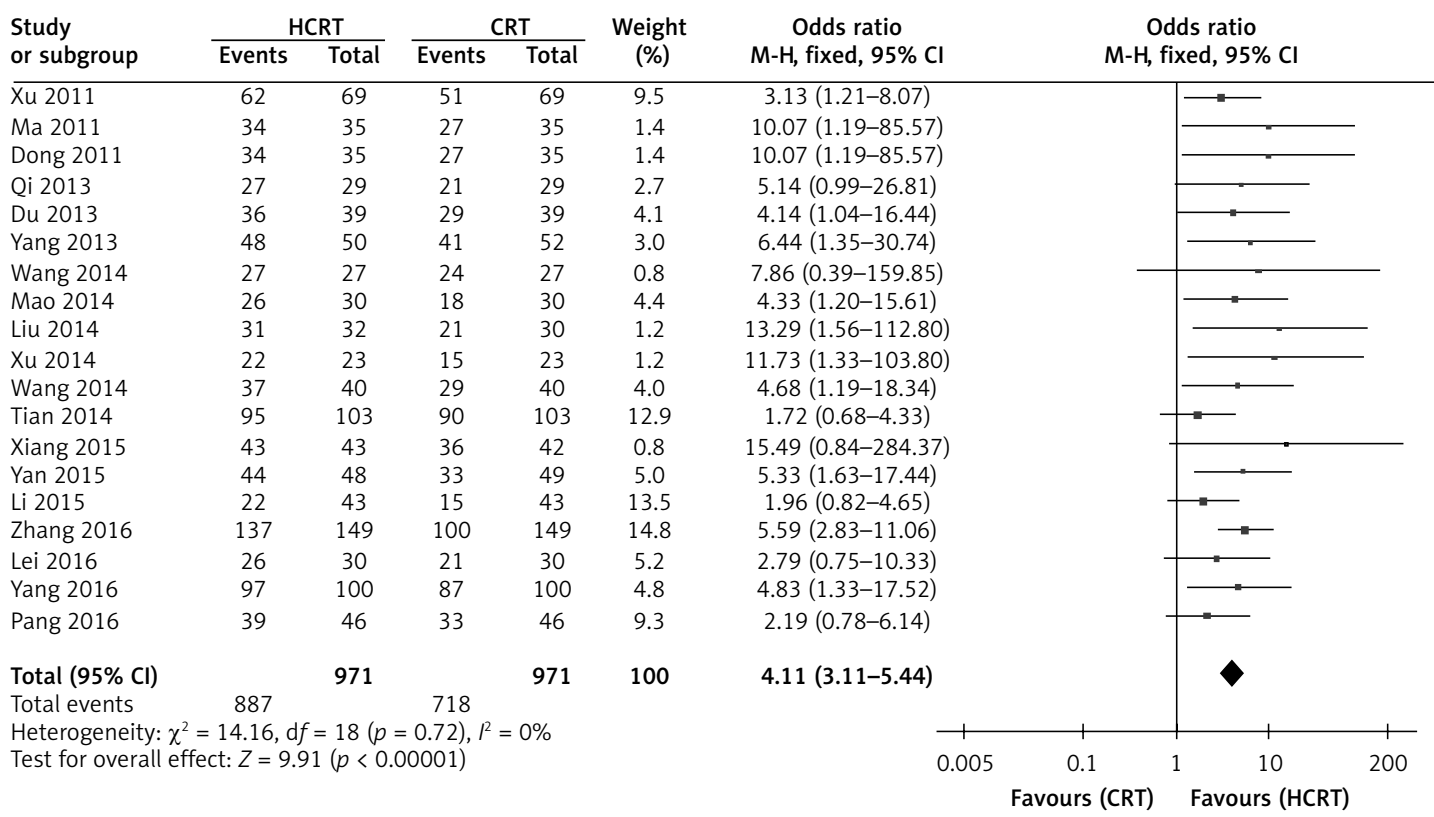

Figure 3. Forest plot for meta-analysis of the overall effective rate (OER) between the CRT group and the HCRT group

suppression and 10 studies reported about bladder problems. The investigations included in this study were without statistical heterogeneity, so the fixed-effect model was used for meta-analysis and the results showed that the CRT and HCRT groups were statistically similar with respect to gastrointestinal disorders, bone marrow suppression and bladder problems (Table II).

\section{Publication bias}

The funnel plot analysis of the overall effective rate showed that the symmetry of the funnel plot was better (Figure 6), which suggested that the results were less likely to be affected by publication bias.

\section{Discussion}

Recently, thermotherapy is widely used in the treatment of advanced cancers and with prospective effect [27]. HT takes advantage of the tumor tissue pathophysiological characteristics that the tumor cells are more sensitive to the heat than normal ones, so when the tumor tissue is heated with a physical temperature of $42.5-43.5^{\circ} \mathrm{C}$ for 60-120 min, the tumor cells will be destroyed, but not the normal tissue. The main anti-tumor mechanisms of HT include: (1) inhibiting the proliferation of tumor cells and inducing apoptosis [28]; (2) reducing the vascular endothelial growth factor synthesis of tumor tissue, thereby undermining and reducing the tumor angiogenesis [29]; (3) stimulating the body's cellular and humoral immune system to improve the body's anti-tumor immune effect [30]; (4) increasing the sensitivity of the tumor tissue to CRT [31]. The published randomized controlled trials (RCTs) mostly are small sample size and single-center studies, and the methods of HT were various, resulting in lower reliability. Therefore, we performed a comparison of CRT alone with CRT plus $\mathrm{HT}$ in this meta-analysis.

This systematic meta-analysis incorporated 19 RCTs which were published in the last 5 years with almost the same method of HT, deep pelvic local hyperthermia, and most of which used the same hyperthermia equipment: an sR-1000type deep RF hyperthermia machine (Shenzhen Advanced Technology Corporation). In addition, extra-cavitary and intra-cavitary radiotherapy and the conventional fractionated radiotherapy were mostly adopted for radiotherapy and cisplatin (DDP) was always used for chemotherapy. Also, all the patients included in this study were Chinese. As a result, it was without methodological or ethnic heterogeneity. The pooled analysis demonstrated that CRT plus HT was associated with a greater 1-year TFSR, CRR, and OER than CRT alone. Moreover, there was no increase in the incidence of adverse reactions. The results also showed that the HCRT group had significantly lower PRR and SDR compared with the CRT group, which was mainly because the incorporated studies mostly had small sample sizes. When the CR cases notably increased in the HCRT group, the number of PR and SD were bound to decrease, thus contributing to lower PRR and SDR in the HCRT group. Although the PRR decreased, the total effective rate still significantly increased in the HCRT group. As the 3-year survival rate between the HCRT group and the CRT group was compared only by three studies, the outcome showed that 


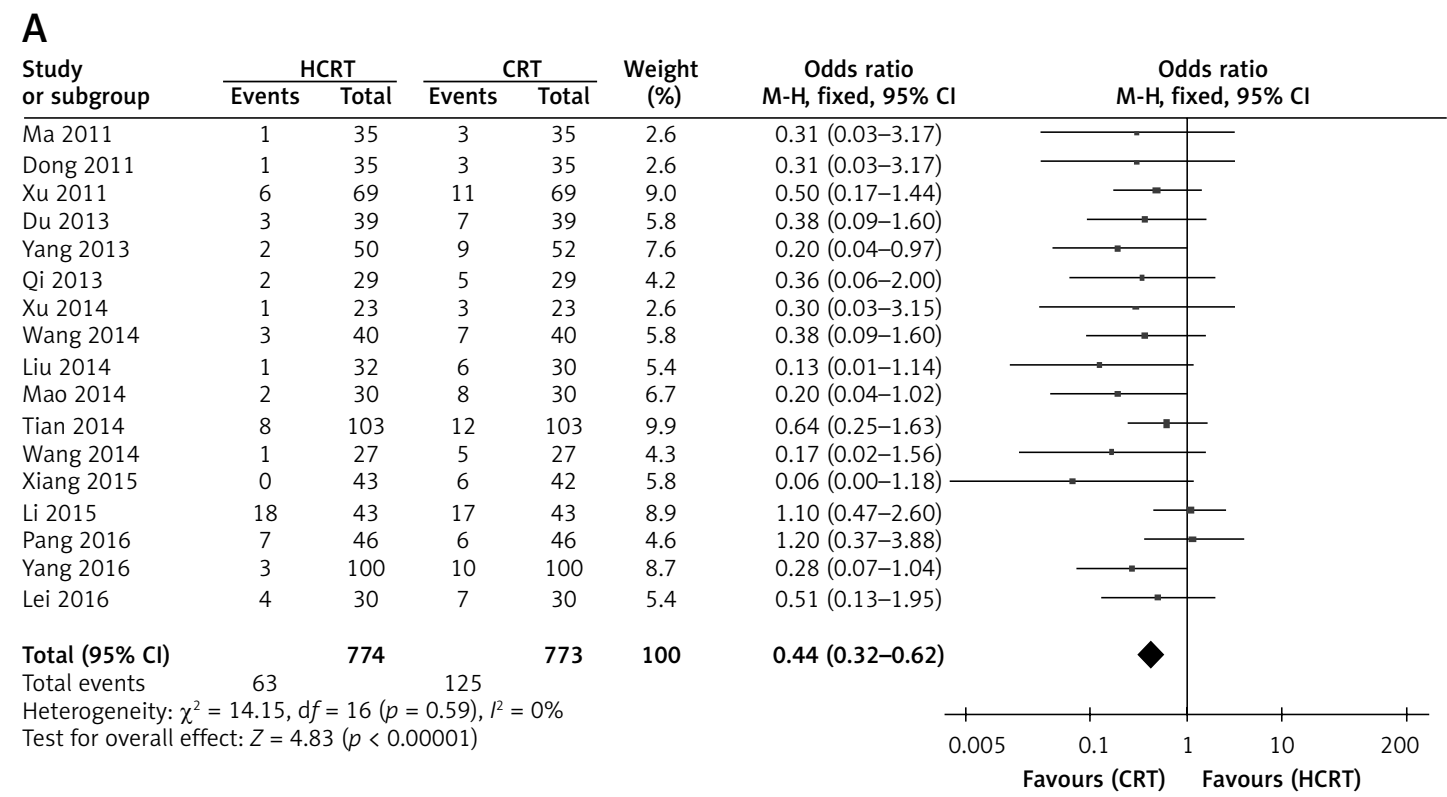

B

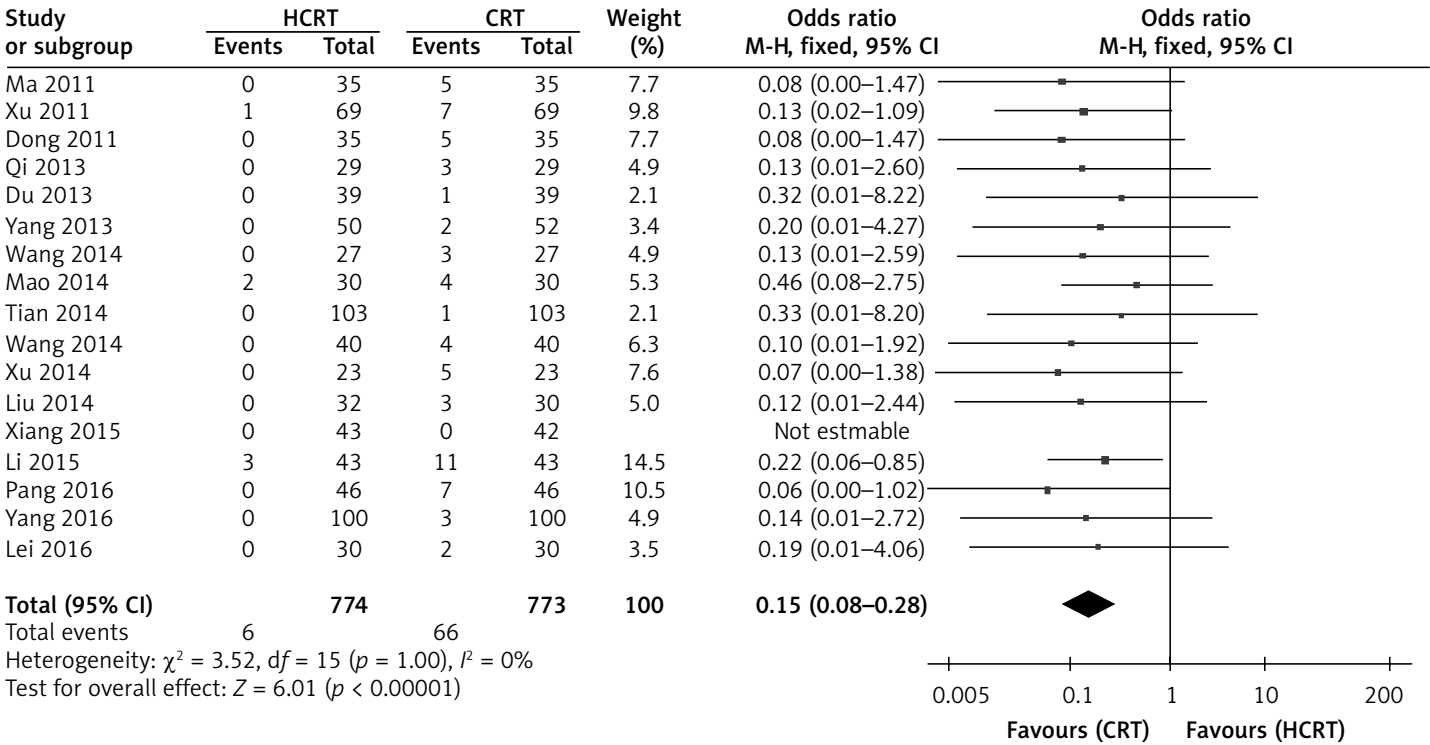

Figure 4. Forest plot for meta-analysis of disease stability rate (SDR (A)) and partial remission rate (PDR (B)) between CRT alone and CRT combined with HT

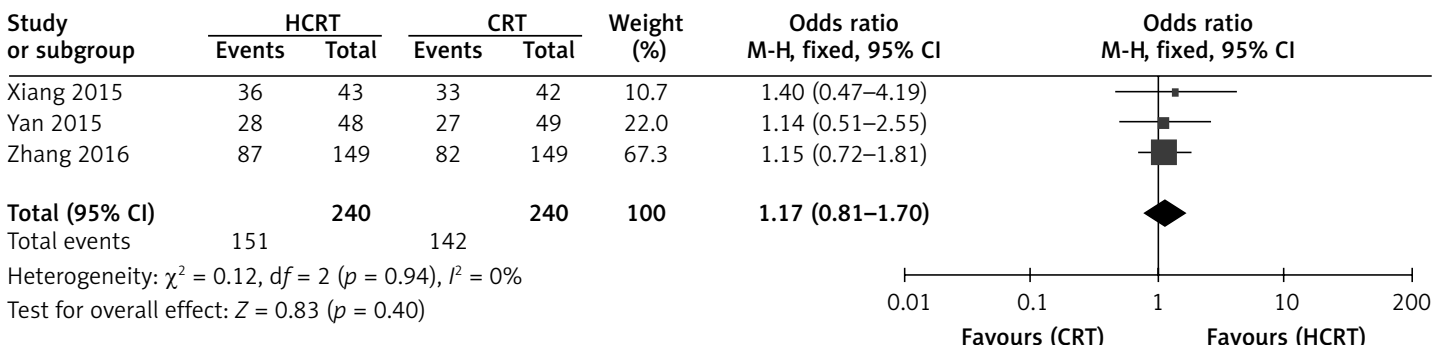

Figure 5. Forest plot for long-term efficacy 3-year SR between the CRT group and the HCRT group

the 3-year survival rate of the HCRT group was similar to that of the CRT group, without statistical significance. In short, radiotherapy and chemotherapy combined with hyperthermia in treat- ment of advanced cervical cancer is a reasonable, effective and safe treatment therapy. However, its long-term efficacy remains to be verified by more large-scale, high-quality RCTs. In addition, improv- 


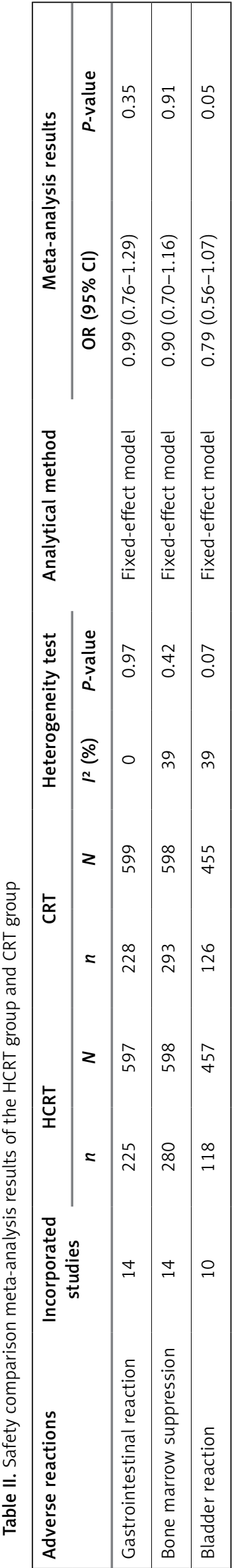

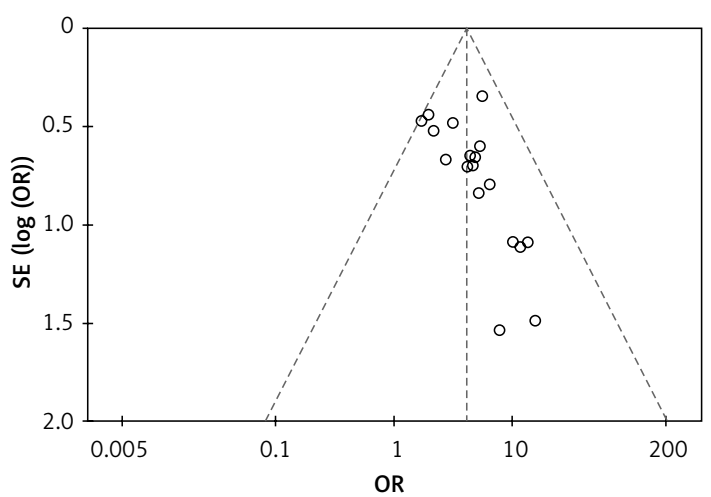

Figure 6. Funnel plot analysis of overall effective rate (OER) between the CRT group and the HCRT group

ing the efficacy as well as reducing toxicity is the most important issue to focus on in the future clinical research.

As HT in clinical practice has not been universally used in western countries, and some HT methods are not consistent with the methods in this study, the included literature was mainly Chinese literature, which may lead to the results of the analysis lacking great significance internationally. On the other hand, because it is advantageous to avoid racial heterogeneity, the results at least in China possess greater clinical significance. Due to the lack of adequate clinical data from the investigations, long-term efficacy of the two groups could not be evaluated exactly, and thus could not achieve comprehensive evaluation of the treatment model of HCRT and CRT, so it is proposed that the clinical trials should increase the observation indicators of long-term efficacy.

In conclusion, HCRT is comprehensive treatment method for cervical cancer and it has a promising future. However, this method remains associated with many problems in clinical practice. These problems include non-destructive testing temperature technology, implementation frequency, duration of HT sessions, the route and dose of radiotherapy, the choice and compatibility of chemotherapy drugs, and the sequence of $\mathrm{HT}$, radiotherapy and chemotherapy. Further research is required to determine the best way to achieve optimal efficacy of HCRT and reduce its side effects.

\section{Conflict of interest}

The authors declare no conflict of interest.

\section{References}

1. Therasse P, Arbuck SG, Eisenhauer EA, et al. New guidelines to evaluate the response to treatment in solid tumors. European Organization for Research and Treatment of Cancer, National Cancer Institute of the United States, National Cancer Institute of Canada. J Natl Cancer Inst 2000; 92: 205-16. 
2. Hanawa S, Mitsuhashi A, Usui H, et al. Daily low-dose cisplatin-based concurrent chemo-radiotherapy for the treatment of cervical cancer in patients 70 years or older. Int J Gynecol Cancer 2015; 25: 891-6.

3. Chen MF, Tseng CJ, Tseng CC. Adjuvant concurrent chemo-radiotherapy with intensity-modulated pelvic radiotherapy after surgery for high-risk, early stage cervical cancer patients. Cancer J 2008; 14: 200-6.

4. Datta NR, Ordóñez SG, Gaipl US, et al. Local hyperthermia combined with radiotherapy and-/or chemotherapy: recent advances and promises for the future. Cancer Treat Rev 2015; 41: 742-53.

5. Crezee J, van Leeuwen CM, Oei AL, et al. Biological modelling of the radiation dose escalation effect of regional hyperthermia in cervical cancer. Radiat Oncol 2016; 11 : 14-6.

6. Westermann A, Mella O, Van Der Zee J, et al. Long-term survival data of triple modality treatment of stage IIBIII-IVA cervical cancer with the combination of radiotherapy, chemotherapy and hyperthermia - an update. Int J Hyperthermia 2012; 28: 549-53.

7. Lutgens LC, Koper PC, Jobsen JJ, et al. Radiation therapy combined with hyperthermia versus cisplatin for locally advanced cervical cancer: results of the randomized RADCHOC trial. Radiother Oncol 2016; 120: 378-82.

8. Pang RR. Clinical observation of hyperthermia combined with cisplatin concurrent chemotherapy in treatment of locally advanced cervical cancer. Chin Count Med Educat 2016; 8: 134-6.

9. Yang XJ, Fan LX, Song JX, Xie Y. Effect of synchronization of thermotherapy and chemo-radiotherapy on cervical cancer in middle and late stage and CD4+T cells dysequilibrium. Mod J Tradit Med 2016; 26: 2871-4.

10. Lei JH, Yu XJ, Yu YD, Di QF, Cao FJ. A clinical observation of short term effects of local thermotherapy combined with chemo-radiotherapy in treatment of stage betab cervical cancer. Mod Oncol 2016; 24: 1441-3.

11. Zhang AY, Yan J, Xu W, Fu J, Yi JF. Clinical observation and nursing intervention of pelvic hyperthermia combined with concurrent chemo-radiotherapy for advanced cervical cancer. Nurs Pract Res 2016; 3: 087-089.

12. Xiang L, Zhong L, Ye H, Zhang ZH, Luo HQ, Wang LY. The clinical observation of the treatment of locally advanced cervical cancer with hyperthermia combined with cisplatin. J Luzhou Med College 2015; 38: 260-3.

13. Li GH. Effect of deep, radiotherapy and concurrent chemo-radiotherapy for locally advanced cervical cancer. Chin J Med Gu 2015; 17: 206-7.

14. Yan J, Fu J, Du JH, Xu FW, Zhang HN. Studies of concurrent chemotherapy combined with pelvic hyperthermia in the treatment of locally advanced cervical cancer effect. Pract Clin Med J 2015; 19: 59-61.

15. Wang P, Hui H, Wu L, Han SX, Wu L. The clinical application of hyperthermia combined with synchronous radiotherapy chemotherapy in locally advanced cervical cancer. Med J West China 2014; 26: 1596-8.

16. Tian XF, Liu AL, Han ZH, Wang J, He QB, Bai LH. Clinical research on the treatment of advanced cervical cancer with hyperthermia combined with radiotherapy and chemotherapy. Shaanxi Med J 2014; 43: 871-3.

17. Wang R, Yang CY. Efficacy of deep hyperthermia combined with radiotherapy chemotherapy on locally advanced cervical cancer. Clin Med 2014; 34: 70-1.

18. Liu XL, Bao YN. Efficiency of deep hyperthermia combined with concurrent chemo-radiotherapy for locally advanced cervical cancer. J Chengdu Med College 2014; 9: 38-40.
19. Mao HH, Zhong Y. Clinical study on endogenetic field tumor hyperthermia combined with chemotherapy and radiotherapy in the treatment of advanced cervical cancer. Chin J Clin Oncol Rehabil 2014; 21: 585-8.

20. Xu MZ, Li XB, Zou YB, Xiong Y, Ding W. Short-term curative effect of concurrent thermotherapy and radiotherapy in the treatment of advanced cervical cancer. Jiangxi Med J 2014; 49: 736-8.

21. Yang CY, Long AE. The short term efficacy of hyperthermia radiotherapy and chemotherapy for advanced cervical cancer patients. Mod Oncol 2013; 21: 385-7.

22. Du K, Jin YQ, Li Q. Clinical study of hyperthermia combined with radio-chemotherapy for the treatment of advanced cervical cancers. Hainan Med J 2013; 24: 1185-6.

23. Qi HX, Du K, Sun QS. Clinical observation of intra-modulation radiation therapy combined with hyperthermia therapy for advanced cervical cancer. J Pract Med 2013; 29: 2928-9.

24. Ma AP, Zhong SJ, Li F, Wang Y. Efficiency of deep hyperthermia combined with concurrent radio-chemotherapy in the treatment of stage betab cervical cancer. Mod Oncol 2011; 19: 1409-11.

25. Xu JL, Wang Y, Ren L, Gao D. Clinical study on deep hyperthermia combined with concurrent radio-chemotherapy for the treatment of advanced cervical cancers. Inner Mongolia Med J 2011; 43: 1045-7.

26. Dong JL, Yao J. The clinical curative effect observation concomitant chemo-radiation combined deep heat treatment for cervical cancer. Adv Biomed Engineer 2011; 32: 233-5.

27. Kok HP, Kotte ANTJ, Crezee J. Planning, optimisation and evaluation of hyperthermia treatments. Int J Hyperthermia 2017; 33: 593-607.

28. Michalakis J, Georgatos SD, Romanos J, et al. Micromolar taxol, with or without hyperthermia, induces mitotic catastrophe and cell necrosis in HeLa cells. Cancer Chemother Pharmacol 2005; 56: 615-22.

29. Sawaji Y, Sato T, Takeuchi A, Hirata M, Ito A. Anti-angiogenic action of hyperthermia by suppressing gene expression and production of tumour-derived vascular endothelial growth factor in vivo and in vitro. Br J Cancer 2002; 86: 1597-603.

30. Frey B, Weiss EM, Rubner $Y$, et al. Old and new facts about hyperthermia-induced modulations of the immune system. Int J Hyperthermia 2012; 28: 528-42.

31. Brüningk SC, ljaz J, Rivens I, Nill S, Ter Haar G, Oelfke U. A comprehensive model for heat-induced radio-sensitisation. Int J Hyperthermia 2017: 1-11. doi: 10.1080/ 02656736.2017.1341059. 